\title{
Original
}

\section{The Process of Accepting Functional Impairments among Male Rectal Cancer Patients after Surgery}

\author{
Masataka Horikoshi ${ }^{1}$ and Tamae Futawatari ${ }^{1}$ \\ 1 Department of Nursing, Gunma University Graduate School of Health Sciences, 3-39-22 Showa-machi, Maebashi, Gunma 371-8514, \\ Japan
}

\begin{abstract}
Background and Aims: Male rectal cancer patients experience postoperative anxiety regarding bowel, urinary, and sexual function impairment. These patients must cope with various physical and psychosocial issues. The purpose of this study was to explain the process of accepting postoperative functional impairments among male rectal cancer patients.

Methods: Fourteen male rectal cancer patients who had undergone surgery 6 or more months beforehand were assessed through semi-structured interviews. Data were analyzed using the grounded theory approach.

Results: Simultaneous bowel, urinary, and sexual dysfunction mutually impeded male rectal cancer patients' activities and negatively affected their self-esteem alongside a feeling of lost masculinity. However, patients learned to understand their condition while exploring coping techniques for these three major impairments on their own terms. Patients adopted one of two coping methods, positive acceptance or acceptance with resignation, depending on support structures and the effectiveness of these established coping methods.

Conclusion: Patients' acceptance of the impairment of three major bodily functions should be characterized as a single structure, rather than focusing on accepting bowel dysfunction alone. Support programs should be structured based on the processes characterized through this study along with patient readiness.
\end{abstract}

\section{Article Information}

\begin{tabular}{l}
\hline Key words: \\
Rectal cancer, \\
Male, \\
Postoperative, \\
Functional impairment, \\
Process \\
\hline Publication history: \\
Received: October 24, 2016 \\
Revised: December 22, 2016 \\
Accepted: December 27, 2016 \\
\hline Corresponding author: \\
Masataka Horikoshi \\
Department of Nursing, Gunma University Graduate \\
School of Health Sciences, 3-39-22 Showa-machi, Maebashi, \\
Gunma 371-8514, Japan \\
Tel: +81-27-220-8803 \\
E-mail: masataka @ gunma-u.ac.jp \\
\hline
\end{tabular}

\section{Introduction}

Both the incidence and mortality rates for rectal cancer in Japan gradually increased up to the early 2000s and have remained flat in recent years. ${ }^{1}$ Surgery to remove polyps is now widely performed due to the greater application of colorectal cancer screening, and it is believed that treatment of these polyps during the pre-lesion stage is effective for preventing cancer progression. Although the rectal cancer incidence in females has remained steady, incidence in males has exhibited an increasing trend, and in 2012, rectal cancer was the fifth most common cancer. In addition, long-term clinical monitoring is necessary, as the 10year relative survival rate for this condition is $60.8 \%$.

Although surgeries to treat rectal cancer are generally performed to minimize the operative field in consideration of patient quality of life (QOL), patients may develop secondary bowel or urinary dysfunction if damage to the pelvic plexus cannot be avoided during surgery, and male patients in particular may develop sexual dysfunction. These three major forms of physiological impairment arising after rectal cancer surgery cause a variety of problems affecting patients' everyday activities and can significantly affect patients psychosocially. As such, although greater attention is now given to patient QOL, and surgical techniques that achieve radical preservation (e.g. preservation of the anal sphincter and autonomic nerves) as well as functional preservation have become the mainstream, functional damage may still occur when lateral lymph nodes are dissected even if bilateral nerve-sparing is 
performed. ${ }^{2}$ Moreover, while urinary dysfunction may be temporary or absent, bowel and sexual dysfunction cannot be avoided with current medical techniques, even when patients undergo surgery designed to preserve physiological function. ${ }^{3}$ There have also been cases in which these impairments do not improve even after long periods. It is also believed that in cases of advanced rectal cancer, curability can be prioritized by balancing curative aspects of surgery and autonomic nerve-sparing.

As a result, even after overcoming the dangers of surgery, male rectal cancer patients must face difficulties in performing everyday activities as well as damage to their body image and sexuality. Although patients can consult with their physician regarding preoperative procedures and complicating conditions, patients first experience the sense of loss when bodily dysfunctions actually manifest. Rather than during the perioperative period, this manifestation occurs after the recovery period, when patients' general condition has normalized. It is during this time that an avenue for consultation regarding QOL improvement becomes necessary. Regarding sexuality in particular, in Japan, overt expressions and discussion are stigmatized due to the influence of various cultural and historical factors. Sexuality is becoming more openly acknowledged in comparison to the past, and while the number of counseling centers and clinics related to sexuality is increasing, the facilities that can meet the needs of this patient group are limited.

Under these circumstances, male rectal cancer patients are predicted to experience anxiety over these three major forms of dysfunction, and to cope with various physical and psychosocial problems in life. Adequate monitoring of patients beyond the current support structure may lead to improvement in patient QOL.

In reviewing the nursing literature related to rectal cancer, although research concerning the development of a scale to evaluate bowel dysfunction arising after anterior resection ${ }^{4}$ as well as research regarding the psychosocial impact of bowel dysfunction and self-care measures taken with respect to these impairments have been conducted in Japan, these studies primarily involved bowel dysfunction arising after lower anterior resection surgery. ${ }^{5,6}$ The international literature includes research concerning the relationships between aspects of the rectal cancer itself (stage or surgical time) and demographic characteristics and sexual functional impairments ${ }^{7}$; verification of the effects of pelvic muscle exercises with respect to bowel dysfunction ${ }^{8}$; and, similar to the domestic literature, research to describe the experience of bowel dysfunction and the measures taken to restore a sense of wellness. ${ }^{9,10}$ However, all of the identified studies focused on bowel dysfunction and abdominal symptoms. Based on the above, we were able to determine that studies comprehensively examining the impact of functional impairments in rectal cancer patients on body image, sexuality, and everyday activities are not currently available, and that assessment of the acceptance process related to these impairments is insufficient. In addition, no research conducted to date has limited this assessment to male rectal cancer patients, who are disproportionately affected by sexual dysfunction among these three major forms of postoperative impairment.

Based on the above, it is necessary to first characterize the types of problems facing male rectal cancer patients following surgery and describe how these patients are coping with these problems. After gaining an understanding of these problems, it is necessary to develop a nursing support program and a scale to quantify outcomes in addition to educational programming for healthcare professionals in order to create a support system for patients. To accomplish these tasks, in addition to understanding of the nature of patients' problems and coping methods, the process of understanding in what sequence these actions arise and how a nursing support system could be incorporated is also necessary.

Therefore, the purpose of this study was to explain the process of accepting postoperative functional impairments among male rectal cancer patients, and to provide suggestions for nursing support.

\section{Methods}

\section{Study design}

The purpose of this study was to describe the process followed by postoperative male rectal cancer patients. From a theoretical perspective, the Grounded Theory Approach ${ }^{11}$ (GTA), a qualitative induction approach, was used. GTA is a theoretical structure for describing specific phenomena and clarifying the precise processes a person is experiencing. In addition, symbolic interactionism ${ }^{12}$ formed the theoretical basis for this study. Symbolic interactionism assumes that humans act toward things on the basis of the meanings that these things hold to them. The experiences of male rectal cancer patients were determined to be suitable as the focus of this study because they go through changes over the course of a process based on patients understanding and on how their actions interact with society.

\section{Participants}

This study targeted male rectal cancer patients who had completed surgery and were receiving outpatient care at the Department of Gastroenterology at Hospital A. The participant recruitment method used in this study involved receiving information concerning participants who were believed to meet the selection criteria in advance from attending physicians who were cooperating with the study and introducing these patients to study researchers in the physician's office. Selection criteria were as follows: favorable postoperative clinical course, 6 or more months after surgery which was stable physical condition, ${ }^{13}$ no metastasis to other organs or lymph nodes, and, if a stoma was placed as part of a surgical procedure, the stoma must 
Table 1 Participant Characteristics

\begin{tabular}{cccccc}
\hline case & $\begin{array}{c}\text { Age } \\
\text { group }\end{array}$ & $\begin{array}{c}\text { Marital } \\
\text { status }\end{array}$ & Job & $\begin{array}{c}\text { Clinical } \\
\text { stage }\end{array}$ & $\begin{array}{c}\text { Surgery type } \\
\text { surgery [stoma reversal] } \\
\text { to interview }\end{array}$ \\
\hline A & 60 & Married & No & II & ISR \& loop ileostomy \\
B & 60 & Married & Yes & I & LAR \& loop ileostomy \\
C & 60 & Married & Yes & III & LAR \\
D & 60 & Married & Yes & I & ISR \\
E & 60 & Married & No & II & LAR \\
F & 40 & Married & No & III A & LAR \\
G & 60 & Married & Yes & I & LAR \\
H & 60 & Married & Yes & III & ISR \& loop ileostomy \\
I & 60 & Married & Yes & III & ISR \& loop ileostomy \\
J & 30 & Married & Yes & I & SLAR \& loop ileostomy \\
K & 40 & Married & Yes & III & ISR \& loop ileostomy \\
L & 60 & Married & Yes & I & SLAR \\
M & 60 & other & Yes & IIIb & LAR \\
N & 60 & Married & Yes & III & SLAR \\
\hline S & Ses & 13 \\
\end{tabular}

Surgery: ISR intersphinctericresection, SLAR Super Low Anterior Resection, LAR Lower anterior resection

have been removed 6 or more months prior. Patients who had undergone minimally invasive transanal surgery (MITAS) or surgery that did not cause autonomic nerve damage were excluded. Fourteen male patients aged between 30-60 years were selected for participation, of whom five had undergone intersphincteric resection (ISR), three had undergone super-low anterior resection (SLAR), and six had undergone lower anterior resection (LAR). Each of the six participants who had undergone LAR had a temporary stoma placed. The time from completion of surgery to interview ranged from 9-27 months. An overview of participants is provided in Table 1 .

\section{Ethical considerations}

This study was approved by the Gunma University Graduate School of Medicine Clinical Research Ethical Review Board (Approval No. 11-9).

Participants were briefed regarding the purpose of this study and the nature of their participation, and also received written and oral explanations of various ethical concerns. Agreement to participate in this study were obtained from all participants. To protect participants' privacy, they were interviewed individually in an outpatient room by a single researcher. Additionally, because the content of interviews sometimes involved intimate details of the participants' lives, an interview was prepared to aid in devising expressions that would minimize the psychological stress experienced by participants.

\section{Data collection}

Data collection was conducted by obtaining information from participants' electronic medical records with their consent (age, occupation, family structure, presence of a life partner, employment status, disease and treatment course, and nursing-related issues encountered), in addition to carrying out semi-structured interviews with participants. Interviews focused on participants' understanding of their physical symptoms and psychological burden from the time of diagnosis until the interview, as well as any psychological changes experienced. Interviewers asked participants to recall chronologically their experiences from the time of diagnosis until the interview, with particular respect to the three major physiological impairments, the problems accompanying their onset, coping techniques employed, and practices that were supportive, as well as changes in their self-esteem, body image, and sexuality. Interview content was recorded with the consent of all participants using a voice recorder. The mean duration of the interviews was $50 \mathrm{~min}$. The data collection period was between August 2011 and August 2014.

\section{Data analysis}

The content of participant interviews was recorded verbatim and then analyzed using the GTA techniques. Specific analytical methods are detailed below.

\section{1 ) Open coding}

After sufficiently reviewing the recordings, they were divided by content, and then subdivided. Properties and dimensions were extracted from each subdivision and labeled based on these divisions. Similar labels were grouped into categories and labeled as such.

\section{2 ) Axial coding}

While evaluating the relationships between categories, the category of focus was left unchanged, while other categories were made subcategories. Categories and subcategories were grouped together depending on their properties and dimensions indicating the characteristic; conditions, contexts, action/interactional strategies, and consequences were prepared as coding paradigms, upon which a diagram was created. The paradigm was a framework to constitute a concept as a process. Data collection was repeated after subsequent- 
ly performing theoretical sampling, i.e., checking an emerging theory against reality by sampling incidents. Data collection and analysis were conducted in an alternating fashion based on the above procedure. The categories and subcategories extracted from the data obtained from each participant were integrated, and a further integrated diagram was created. In addition, a story line based on this integrated diagram was created to explain various phenomena. While conducting investigations and analysis simultaneously, two additional investigations were conducted when researchers determined that new data could not be confirmed, and the investigations were considered complete when theoretical saturation was confirmed. In this process, no conspicuous difference across age groups and marital status was observed.

To ensure the reliability of the analysis results, assessments were conducted under the supervision of multiple researchers who were proficient in qualitative research practices. Additionally, researchers who conducted interviews subsequently confirmed the content obtained, improving its veracity by obtaining approval for the content.

In this paper, categories are marked using [ ], subcategories are marked using $《\rangle$, labels are marked using $\langle>$, and interview data are marked using “"”.

\section{Results}

As a result of the analysis of the experiences and coping measures of postoperative male rectal cancer patients presenting with impairment of three major bodily functions, [Developing methods to cope with impairment of three major bodily functions] served as the category of focus, and 12 subcategories were extracted as related phenomena, including: 《Start of bowel dysfunction》, 《Understanding of the reality of urinary dysfunction》, 《Initial coping with bowel dysfunction》, 《Uncontrollable defecation》, 《Negative effects caused by bowel dysfunction》, 《Understanding of the reality of bowel dysfunction》, 《Understanding of the reality of sexual dysfunction》, 《Damage to self-esteem》, 《Regret regarding surgery》, 《Actual feeling of receiving support》, 《Acceptance with resignation》, 《Positive acceptance》. Category, subcategory, and labels were displayed in Table 2. In addition, Fig. 1 was also an integrated diagram displaying the relationships between categories and subcategories.

The following sections explained the story line, categories, and subcategories.

\section{1) Story line}

Male rectal cancer patients experience the $《$ start of bowel dysfunction》 shortly after surgery, and feel anxiety about their condition during a healing process

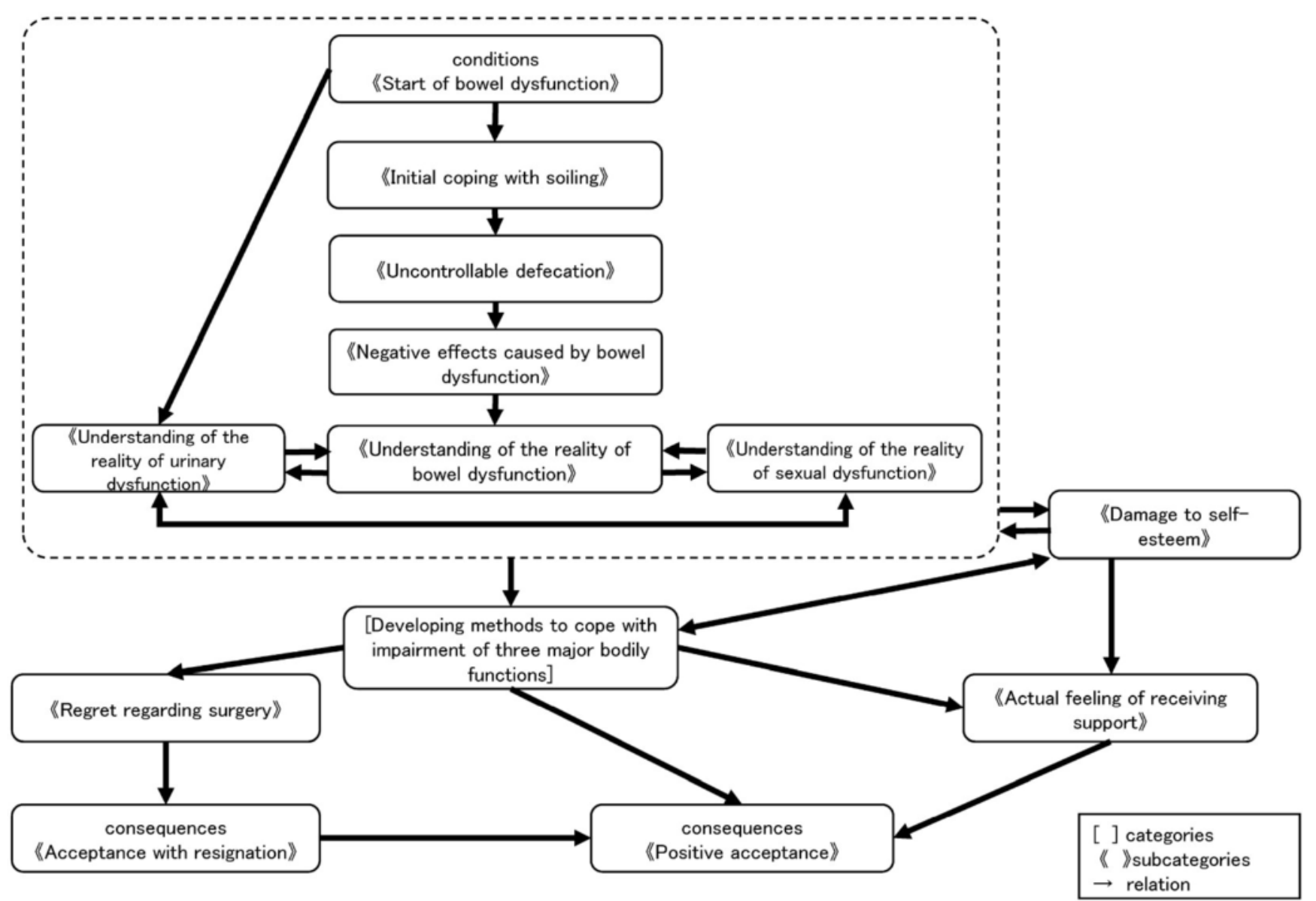

Fig. 1 The Process of accepting of functional impairments among male rectal cancer patients after surgery 
Table 2 Construct of the process

\begin{tabular}{|c|c|c|}
\hline paradigms & [category], 《subcategories》 & $\langle$ labels〉 \\
\hline conditions & 《Start of bowel dysfunction》 & $\begin{array}{l}\text { postoperative constipation } \\
\text { soiling that occurs immediately after surgery } \\
\text { diarrhea that occurs immediately after surgery } \\
\text { mixed urine and stool } \\
\text { postoperative sudden bowel movement urges }\end{array}$ \\
\hline \multirow[t]{10}{*}{$\begin{array}{l}\text { action/interactional } \\
\text { strategies }\end{array}$} & 《Understanding of the reality of urinary dysfunction》 & $\begin{array}{l}\text { difficulty urinating } \\
\text { frequent urge to urinate } \\
\text { discomfort while urinating } \\
\text { feeling of urinary incontinence }\end{array}$ \\
\hline & 《Initial coping with soiling》 & $\begin{array}{l}\text { wear diapers to manage soiling } \\
\text { wear pads to manage soiling } \\
\text { change underwear dirtied by soiling }\end{array}$ \\
\hline & 《Uncontrollable defecation》 & $\begin{array}{l}\text { irregular stool shape and texture } \\
\text { elusive urge to defecate } \\
\text { unpredictable diarrhea } \\
\text { slow urge to defecate even now } \\
\text { unbearable flatulence } \\
\text { severe bowel dysfunction beyond expectations }\end{array}$ \\
\hline & 《Negative effects caused by bowel dysfunction》 & $\begin{array}{l}\text { outdoor activities negatively impacted by soiling } \\
\text { inability to engage in hobbies due to soiling } \\
\text { occupation negatively impacted due to flatulence } \\
\text { occupation negatively impacted due to soiling } \\
\text { severe anal chafing } \\
\text { increased number of restroom visits due to concerns over soiling } \\
\text { dilution of social relationships } \\
\text { difficulty varying routine } \\
\text { difficulty engaging in sexual activity due to soiling }\end{array}$ \\
\hline & 《Understanding of the reality of bowel dysfunction》 & $\begin{array}{l}\text { understanding of current soiling condition } \\
\text { reduced number of bowel movements due to constipation } \\
\text { grasp of stimuli that cause diarrhea }\end{array}$ \\
\hline & 《Understanding of the reality of sexual dysfunction》 & $\begin{array}{l}\text { erectile dysfunction } \\
\text { dulled erogenous sensation } \\
\text { dulled ejaculation sensation } \\
\text { incomplete ejaculation }\end{array}$ \\
\hline & 《Damage to self-esteem》 & $\begin{array}{l}\text { self-pity accompanying sexual dysfunction } \\
\text { sense of resistance against wearing a diaper } \\
\text { self-pity due to urinary incontinence } \\
\text { self-pity accompanying soiling }\end{array}$ \\
\hline & $\begin{array}{l}\text { [Developing methods to cope with impairment of three } \\
\text { major bodily functions] }\end{array}$ & $\begin{array}{l}\text { regulating bowel movements with drug therapy } \\
\text { planning the timing of bowel movements } \\
\text { strengthening the anal sphincter to improve soiling } \\
\text { coordinating meals } \\
\text { planning urinating methods } \\
\text { managing anal chafing } \\
\text { changing underwear to manage soiling } \\
\text { keeping their abdomen warm } \\
\text { changing their routines } \\
\text { planning outdoor activities } \\
\text { resisting bowel movement urges }\end{array}$ \\
\hline & 《Regret regarding surgery》 & $\begin{array}{l}\text { fear of worse soiling in the future } \\
\text { doubts over whether they should have a stoma opened } \\
\text { unease concerning recurrence } \\
\text { great desire to see improvement in their bowel dysfunction }\end{array}$ \\
\hline & 《Actual feeling of receiving support》 & $\begin{array}{l}\text { spousal support } \\
\text { healthcare provider support } \\
\text { support at work } \\
\text { gratitude for family and friends' understanding } \\
\text { social networking service (SNS) gratitude }\end{array}$ \\
\hline \multirow[t]{2}{*}{ consequences } & 《acceptance with resignation》 & $\begin{array}{l}\text { forcing oneself to accept the status quo } \\
\text { giving up on seeing improvement in their soiling } \\
\text { accepting the possibility of recurrence or metastasis } \\
\text { giving up improvement of their sexual condition }\end{array}$ \\
\hline & 《Positive acceptance》 & $\begin{array}{l}\text { clear explanation of soiling } \\
\text { well-stated description of their relationship with their bodies } \\
\text { relief due to avoiding having a stoma opened }\end{array}$ \\
\hline
\end{tabular}

Category is marked using [] , subcategories are marked using 《》

comprised of experiencing pain and poor physical condition. These patients also experience urinary incontinence, frequent urination, and urges to urinate during this period, and although patients are some- times able to develop an understanding of the reality of urinary dysfunction $》$, the presence of defecation and urination occurring simultaneously due to fecal leakage (hereinafter, "soiling") and frequent bowel move- 
ments cause patients to focus on bowel dysfunction first. The use of adult diapers or incontinence pads to manage soiling, in addition to frequently rushing to use the restroom, describe patients'《initial coping with bowel dysfunction》. Even after being discharged from the hospital, they continue to feel anxiety due to 《uncontrollable defecation》. Their jobs, hobbies, and other activities are negatively impacted by soiling and other symptoms of bowel dysfunction that occur each day, leaving them helpless in the face of 《negative effects caused by bowel dysfunction》. These patients notice during these experiences that they do not feel an urge to defecate and become accustomed to the intervals of their bowel movements. Moreover, these patients gain an 《understanding of the reality of bowel dysfunction》as they learn the changes to the nature of their stool and the impact of their activities and different foods they consume.

As these patients begin to gain 《understanding of the reality of bowel dysfunction》, their perspectives broaden slightly. After surgery, these patients also notice the onset of erectile dysfunction or complete inability to achieve an erection, in addition to decreasing interest in the opposite sex. Additionally, these patients face numerous concerns at once, such as the fear that they will have a soiling incident during sexual activity or will be unable to achieve or maintain an erection, or that they will have a soiling incident while using the restroom before such an activity. These patients are further humiliated by their need to use adult diapers or incontinence pads and by observing the burdens of partner after repeated incidents. These patients also feel a loss of their masculinity, resulting in continual significant 《damage to self-esteem》. This kind of 《damage to self-esteem》can further interact with patients' impairments. Therefore, these patients can repeatedly suffer painful experiences while deepening their understanding of their condition, increasing their 《damage to self-esteem》. However, as they start to gain the ability to cope in ways that suit them, these patients progress towards [developing methods to cope with impairment of three major bodily functions]. To date, these patients have not only maintained and updated their coping methods, but have gained a sense of control through self-management measures such as medicinally regulating their bowel movements and taking steps to prevent anal chafing, and by reducing the 《damage to self-esteem》suffered, they become able to exhibit 《positive acceptance》 of impairment of the three major bodily functions. However, if these coping methods yield inadequate results, these patients' concerns about cancer recurrence or metastasis will be reinforced, and the feeling that having a colostomy would have been better and denial of present conditions will be strengthened. Although in some cases patients will begin to feel 《regret regarding surgery》, these patients will later begin to believe their situation is hopeless, and will exhibit 《acceptance with resignation》 of their condition.

In addition, the spouses, friends, and others close to these patients will gain understanding of their impairments, allowing patients to experience an 《actual feeling of receiving support》 by [developing methods to cope with impairment of three major bodily functions], strengthening their capacity to achieve 《positive acceptance》 of their conditions. By strengthening these patients' 《actual feelings of receiving support》, 《acceptance with resignation》 can be improved to $《$ positive acceptance》.

\section{2 ) Categories and subcategories}

\section{(1) Start of bowel dysfunction}

《Start of bowel dysfunction》describes patients' first experience with bowel dysfunction shortly after surgery and the start of their anxious psychological state. Of their three major impairments, male rectal cancer patients first experience bowel dysfunction, including a variety of symptoms such as $<$ postoperative constipation〉, 〈soiling that occurs immediately after surgery $\rangle$, 〈diarrhea that occurs immediately after surgery $\rangle$, 〈mixed urine and stool〉, and 〈postoperative sudden bowel movement urges $>$, which appear when postoperative healing ceases to progress.

"I couldn't control [my bowel movements] after my surgery so I had to wear pads and diapers, there were many times when I would just suddenly go." (Mr. J)

"I had watery diarrhea the first time I went after my surgery. I could only feel it right as it was coming out, so I had diapers bought for me and immediately wore one. After that, my stool was soft like diarrhea and felt like it wasn't solid. I could feel it, but again, only right as it was coming out." (Mr. K)

(2) Understanding of the reality of urinary dysfunction

《Understanding of the reality of urinary dysfunction》 describes patients' understanding of urinary dysfunction, such as 〈difficulty urinating $\rangle$, 〈frequent urge to urinate $\rangle$, 〈discomfort while urinating $\rangle$, and $\langle$ feeling of urinary incontinence $\rangle$. Although some patients developed a grasp of their condition a few days after removal of the urethral catheter and others did not fully notice it, there were additional patients who gained understanding after becoming used to their bowel dysfunction symptoms. While no patients exhibited urinary dysfunction severe enough to cause significant leakage or inability to urinate naturally, all experienced discomfort.

"I couldn't pee at all for a few days after my surgery. Even now, there is less flow. After I go once, it does not take me an hour I feel like going again. It gives me a weird feeling. Maybe it's leftover urine, or rather the flow is weak, my sensations are weak too." (Mr. K)

“At first I couldn't urinate. I didn't know if my urethra was damaged by the tube during surgery, but something felt funny." (Mr. C)

(3) Initial coping with soiling

《Initial coping with soiling》 describes how 
patients first choose to deal with soiling arising immediately after surgery. Patients cannot rush to the toilet because their activity is reduced following surgery. In addition, soiling does not improve over time, and patients have no choice but to <wear diapers to manage soiling , 〈wear pads to manage soiling〉, or 〈change underwear dirtied by soiling $\rangle$.

"I sleep wearing a pad when my stool is soft. I haven't had much leakage lately and on some days I pass only a small amount, but I still wear a pad just in case." (Mr. I)

"I haven't stopped wearing a pad for urine leakage since my surgery. My workspace is a bit far from a restroom so I have a feeling that I will have leaks in the time it takes me to get there." (Mr. H)

\section{(4) Uncontrollable defecation}

《Uncontrollable defecation》describes the condition of difficulty in controlling bowel movements that had never been experienced before the operation. In cases of diarrhea, patients can experience different urges, such as being unable to hold their movement until reaching the toilet, having no sensation while defecating, and defecating unknowingly. Male rectal cancer patients continued to feel anxiety concerning various symptoms of uncontrollable defecation, such as $\langle$ irregular stool shape and texture $\rangle$, 〈elusive urge to defecate $\rangle$, 〈unpredictable diarrhea〉, 〈slow urge to defecate even now $\rangle$, 〈unbearable flatulence $\rangle$, and $\langle$ severe bowel dysfunction beyond expectations $\rangle$, and lost their sense of control.

In addition, these patients noticed their urinary dysfunction and urinary flow difficulties while urinating, and instances of mixed effects such as soiling incidents were also observed.

"I go to the toilet much less often now-it is painful. I also go in the middle of the night too. When I need to go at night, I usually have to keep going until morning. After dinner, I need to go about every 20 minutes until around 11 P.M." (Mr. A)

"I go a lot at night. When I start going, I may say it does not stop. I pass a small amount each time. I will go once, and then 5 or 6 minutes later I will need to go again. I don't usually go during the day. Usually only once or so even if I went." (Mr. M)

"I have peed while standing about 2-3 times recently, but I usually do so while sitting. I do so because I notice that I have difficulty with flow, and also because I worry that I will suddenly have a bowel movement." (Mr. G)

(5) Negative effects caused by bowel dysfunction

《Negative effects caused by bowel dysfunction》 describes how patients' everyday activities are negatively impacted by bowel dysfunction. Soiling and flatulencerelated problems such as <outdoor activities negatively impacted by soiling , 〈inability to engage in hobbies due to soiling , 〈occupation negatively impacted due to flatulence〉, and 〈occupation negatively impacted due to soiling $>$ could limit patients' activities. Secon- dary problems such as 〈severe anal chafing〉, 〈increased number of restroom visits due to concerns over soiling 〉, 〈dilution of social relationships〉, and 〈difficulty varying routine $\rangle$ could also occur. However, problems such as 〈difficulty engaging in sexual activity due to soiling $>$ were facilitated by patients' anxiety over their bowel dysfunction during sexual activity. In this manner, male rectal cancer patients were swayed by the effects of bowel dysfunction daily.

"I have to go often so I don't go far [from a toilet]. At most, I'll go about 40 minutes away. I worry about the urge to go. The place where I'm headed for a trip probably has a toilet, but once I go to the toilet I will need to go again about 6 or 7 more times later. I love to travel but I can't go anymore." (Mr. G)

"It's a big problem if I start wanting to go to the toilet in the middle of sex. [OMISSION] In the end, I don't think I'll be able to do it like I did in the old days." (Mr. K)

"I don't have a job. I quit after I got sick. I figured I couldn't continue the way I was." (Mr. L)

(6) Understanding of the reality of bowel dysfunction

《Understanding of the reality of bowel dysfunction》describes patients' understanding of both unstable bowel movements and timing, such as 〈understanding of current soiling condition $\rangle$ and $\langle$ reduced number of bowel movements due to constipation>, as well as their understanding of the impact of food and activities on the shape and texture of their stool, indicated by their 〈grasp of stimuli that cause diarrhea〉. For better or worse, some patients' bowel dysfunction stabilized, and others prepared to accept their impairment.

"With my current condition, I have leakage even if I don't apply any pressure. But, I have a good grasp of the timing and pattern of my bowel movements." (Mr. A)

"After I had alcohol or when I step out of my bath and $I$ am in a state nearly naked because the bath is hot, my stomach gets cold, it makes me have diarrhea. So long as I don't have that sensation, I don't often have diarrhea." (Mr. J)

(7) Understanding of the reality of sexual dysfunction

《Understanding of the reality of sexual dysfunction》 describes patients' recognition of the sexual impairments that had already manifested, after they broaden their perspective as a result of gaining 《understanding of the reality of bowel dysfunction》. Male rectal cancer patients experience clear declines in sexual function, as exhibited by 〈erectile dysfunction〉, $\langle$ dulled erogenous sensation $\rangle$, 〈dulled ejaculation sensation〉, and 〈incomplete ejaculation〉, compared with the preoperative period in addition to decreasing interest in the opposite sex.

"My sexual condition hasn't changed much from before my surgery. I can get erections. But, I cannot ejaculate. I can get an erection, and reach an orgasm, but it ends there." (Mr. I) 
"I didn't want to make an approach even when I saw a woman. Even if she was pretty, I didn't feel anything. Since my male capacities were gone, I didn' t have sexual feelings anymore. I just lost interest." (Mr. K)

\section{(8) Damage to self-esteem}

《Damage to self-esteem》describes the emotions experienced by patients while being impacted by their impairments each day. As evidenced by feelings such as 〈self-pity accompanying sexual dysfunction〉, 〈sense of resistance against wearing a diaper $\rangle$, 〈self-pity due to urinary incontinence〉, and 〈self-pity accompanying soiling $>$, patients experienced humiliation and shamed from soiling and urine leakage incidents and wearing diapers or pads, as well as due to sexual problems such as erectile dysfunction. Their self-esteem was greatly injured by witnessing the burden placed on those close to them. Additionally, these patients' often experienced a feeling of lost masculinity due to their impairments, and some patients' psychological state did not match the changes to their body image.

"My libido has also decreased. It can be empty. That sensation is important for men, or rather it is because men feel it. Because I don't get them (erections) anymore, I might as well not even be a man anymore." (Mr. B)

"People may tell me I should just wear a diaper, and I have bought diapers too, but I resist wearing them, and they just sit there. Babies and grandpas are the same, you know." (Mr. F)

(9) Developing methods to cope with impairment of three major bodily functions

[Developing methods to cope with impairment of three major bodily functions] is the critical core concept of the process by which male rectal cancer patients come to accept their condition. By continuing to explore coping methods, patients came to better understand their true condition and, as a result, could regain a sense of control. This progress was tied to patients' implementation regarding suitable coping methods to adopt. Patients adopt various coping measures based on their needs, such as <regulating bowel movements with drug therapy>, 〈planning the timing of bowel movements〉, 〈strengthening the anal sphincter to improve soiling $\rangle$, 〈coordinating meals〉, 〈planning urinating methods $\rangle$, 〈managing anal chafing $\rangle$, 〈changing underwear to manage soiling $\rangle$, 〈keeping their abdomen warm $\rangle$, 〈changing their routines $\rangle$, 〈planning outdoor activities〉, and 〈resisting bowel movement urges $>$.

"Now, whenever I go out for a long time, I take anti-diarrheal medicine." (Mr. B)

"Although it's hot, I now pay attention [to temperature] because I am told that it's not good if my stomach gets too cold as it can disrupt my intestinal movements." (Mr. C)

"When my bowel movements get bad I cannot leave my house. I go to a place like a supermarket when I am out because they usually have toilets. Whenever I go anywhere, the first thing I look for is the restroom." (Mr. L)

\section{(10) Regret regarding surgery}

《Regret regarding surgery》 describes patients' regrets expressed over their surgeries due to the lack of an outlet to direct their feelings and helplessness caused by impairments experienced afterward. Patients experienced unease regarding cancer recurrence or metastasis, indicated by <fear of worse soiling in the future , 〈doubts over whether they should have a stoma opened $\rangle$, 〈unease concerning recurrence $\rangle$, and $\langle$ great desire to see improvement in their bowel dysfunction $\rangle$, in addition to greater feelings of doubt concerning the superiority of colostomy. These regrets were also reinforced when patients felt that their coping methods did not yield sufficient results.

"They have colostomy now, right? I heard from my friend that they are more comfortable, but if I get one, I will be considered to be a disabled person. Is it better than what I'm dealing with now?" (Mr. L)

"Now, I just wonder when I will have normal bowel movements. When I go, I widen my stance and squeeze out each bit-I wonder how those muscles will ever come back. I think about this all the time." (Mr. N)

\section{(11) Actual feeling of receiving support}

《Actual feeling of receiving support》describes patients' recognition of how those close to them, such as their spouses or friends, gain understanding of their impairments through activities such as 〈spousal support〉, 〈healthcare provider support〉, and 〈support at work $\rangle$, and patients' psychological well-being gained while expressing 〈gratitude for family and friends' understanding >. In addition, as indicated by 〈social networking service (SNS) gratitude>, some patients gained support from not only friends and family members' understanding, but also from others with similar conditions through internet communication.

"Who supports me? That is my family. My wife supports me in a big way. She helps me cope, and gives me advice, and even listens to me vent my frustrations too." (Mr. L)

"There is a lot to do at work and I have to go, but when my bowel movements won't stop and I can't go, I have someone cover for me. I am grateful for that." (Mr. N)

\section{(12) Acceptance with resignation}

《Acceptance with resignation》 describes the acceptance some patients reach by considering their condition to be hopeless and giving up because they perceive no improvements over time. Various forms of giving up were observed, such as 〈forcing oneself to accept the status quo〉, 〈giving up on seeing improvement in their soiling , 〈accepting the possibility of recurrence or metastasis〉, and 〈giving up improvement of their sexual condition $\rangle$, and not all were negative. 
"Of course if my bowel movements will calm down, I will be able to do a lot more things $\cdots$ With my condition as it is now, I don't think it will get better even over time. My doctor also said that my bowel movements didn't seem to be steadying, and that we would have to wait and see what happens in the future." (Mr. B)

"All that's left is to just wait for my time. There is no point in worrying about metastasis or recurrence, so I don't." (Mr. H)

"I thought that I would heal if I had surgery, but that wasn't the case. My bowel movements haven't gone back to normal, but I just change my underwear if I soil them-that's really all I can do.” (Mr. M)

\section{(13) Positive acceptance}

《Positive acceptance》describes patients' positive attitude regarding their condition gained through living with their impairments, as a result of aspects such as a 〈clear explanation of soiling $>$ and a 〈well-stated description of their relationship with their bodies $\rangle$. Patients gained a sense of satisfaction by developing a system of self-management to cope by continuing to plan their meals and bowel movements, taking steps to prevent anal chafing, and updating their methods if a better alternative was identified. In addition, compared with patients who had stomas opened, some considered their current condition as better than having had a stoma, giving rise to the positive outlook of $\langle$ relief due to avoiding having a stoma opened $>$.

"I have so many constraints now, but compared to right after my surgery, I am much better able to manage things." (Mr. D)

"My chronic need to go is gradually easing and I have leaks less frequently. Maybe I am getting a bit better." (Mr. N)

\section{Discussion}

This study characterized the problems experienced by postoperative male rectal cancer patients caused by the impairment of three major bodily functions as well as the process by which these patients develop and accept coping methods. As a result, even though rectal cancer patients were swayed by bowel dysfunction and a feeling of loss of control, various prior studies ${ }^{9,14-16}$ had obtained results suggesting that by developing coping methods, these patients could reclaim a sense of wellness. However, unlike numerous prior studies that address only coping methods and acceptance of bowel dysfunction, this study newly demonstrated the harsh circumstances faced by male rectal cancer patients who could not develop an understanding of the impairment of three major bodily functions and methods to cope, who were then unable to accept the condition of their bodies.

We propose two reasons for this phenomenon based on the results obtained through this study. First was the exacerbation of sexual dysfunctions in these patients due to their bowel dysfunction as well as the combined impact of their other impairments, as evidenced by their statements such as, "It's a big problem if I start wanting to go to the toilet in the middle of sex. [An omission] In the end, I don't think I'll be able to do it like I did in the old days" caused by <difficulty engaging in sexual activity due to soiling $>$. Another reason is that as these patients' understanding of the reality of their impairments deepens, they begin to feel a sense of lost masculinity, which causes them pain due to increased 《damage to self-esteem》. Gaining confidence with regard to their coping techniques in particular leads to the establishment of coping methods and ultimately acceptance of their impairments.

Based on the above, these observations suggested the necessity for these patients to reach acceptance of their three impairments together, rather than focusing on the process leading to acceptance of their bowel dysfunction only.

To this end, we conducted an assessment of nursing support measures while interpreting the process by which these patients became able to accept their condition based on the core concept of [developing methods to cope with impairment of three major bodily functions].

1 ) Structure of the process leading to [developing methods to cope with impairment of three major bodily functions] and acceptance

In developing methods to cope with their impairment of these three major bodily functions, male rectal cancer patients must come to understand their condition while suffering each day and by trying different coping techniques through trial and error. Because bowel dysfunction occurs shortly after surgery, these patients must quickly learn to cope with the primary symptoms of this condition, such as soiling and sudden bowel movements. However, because it is certainly not possible for these patients to develop coping methods during the approximately 1 -week period spent in the hospital; they must continue to struggle with their conditions on their own following discharge. Moreover, these patients continue to sustain 《damage to self-esteem》during this process due to the stigma attached to the humiliation of soiling and other aspects of bowel dysfunction. ${ }^{15}$ However, because urinary and sexual dysfunction generally do not impact patients' everyday lives, patients will attempt to hide the more prominent bowel dysfunction and focus on these conditions later on. Yet, male rectal cancer patients' recognition of their bowel dysfunction will gradually surface as they become able to cope, and with the compounded effects of their other impairments, these patients will feel helpless. According to Maeda et al. ${ }^{16}$, after pelvic evisceration, rectal cancer patients' efforts to overcome various feelings of loss they experience, such as the loss of organs and the feeling of a loss of continence, are part of the process by which these patients gain a positive attitude about their condition. The three subcategories of 《understanding of bowel dysfunction》, 《understanding of urinary dysfunction》, 
and 《understanding of sexual dysfunction》represent how patients can specifically describe the nature of the conditions affecting their bodies. Patients' readiness to cope with their conditions is indicated by their understanding of the nature of their problems and their progress toward finding solutions. In essence, the male rectal cancer patients targeted in this study overcame loss experiences related to not only bowel dysfunction but also impairment of three major bodily functions, and underwent a process of reclaiming a positive attitude regarding their conditions. As a result, these patients [developed methods to cope with impairment of three major bodily functions]. However, the process of developing coping methods to accomplish this task is insufficient, due to the pain experienced as patients' 《damage to self-esteem》increases as their understanding of their condition deepens. In addition, these patients also experience 《damage to self-esteem》in the form of a sense of loss of their masculinity due to their condition, as evidenced by statements such as, "A man who can't get an erection might as well not be a man" and, "I wear diapers like a baby." As such, patients who cannot resolve the multifaceted issue of learning to understand their three impairments and developing coping methods will be unable to progress to the next step of acceptance of their condition.

After patients [develop methods to cope with impairment of three major bodily functions], differences between patients can appear regarding how they achieve acceptance depending on the effectiveness of these methods and their support structure. If coping methods have low efficacy, or if patients are unable to perceive any improvement, their regrets concerning the treatment process will become stronger. Patients' selfesteem can decline 2 years after surgery and thereafter as their bowel dysfunction continues to deteriorate, ${ }^{17}$ until they finally reach acceptance by giving up on being upset about their condition. Patients often spend increasing amounts of time in their homes in cases like this, leading to decreased activity and dilution of social relationships, which negatively impact their QOL. As QOL is based on patients' individual values and preferences, this behavior cannot be considered negative categorically. However, there is a possibility that secondary health issues may arise from having increasingly insular activities. By contrast, if patients are able to recover a sense of wellness, it can have a positive impact on improvements in their condition, allowing them to become able to manage their negative feelings, ${ }^{10}$ and as such, it is necessary to guide patients toward 《positive acceptance》 as much as possible. These patients must obtain a new sense of control by gaining confidence that they are applying appropriate coping methods while learning to understand the reality of their condition. In addition, 《actual feeling of being supported》was extracted as a concept for strengthening patients' 《positive acceptance》. Prior studies characterized the aspects of the support received by rectal cancer patients as they rebuilt their lifestyles, ${ }^{16}$ and their results are consistent with this study. Stated differently, even in situations where the effects of coping methods cannot not be observed, patients devoted effort to developing these methods, and a supportive presence during this process makes it possible for patients to achieve positive acceptance of their condition.

\section{2 ) Implications for nursing}

In order for male rectal cancer patients to accept their condition after surgery, [developing methods to cope with impairment of three major bodily functions] is a critical factor. Although the presence of nursing support that considers these patients' lifestyles following discharge is important. However, conventional forms of nursing support will likely continue to be implemented, because the degree of each functional impairment depends on the individual, and prior assumption is difficult. Thus, gradually adjusted support is necessary.

An important aspect after providing support is to determine whether care providers are gaining a deeper understanding of patients' impairments. Caregivers should first promote better understanding of the reality of the patient's condition, and then provide information and methodological advice after identifying the coping techniques that best fit that patient. In addition, it is also necessary to provide psychiatric support to these patients, in consideration of how they can experience greater damage to their self-esteem as they deepen their understanding of their condition. Initially, patients should be encouraged to express their feelings, then to learn to assess even difficult situations on their own. Ensuring that patients are able to cope with their circumstances themselves can reinforce their sense of control. To achieve this, conducting assessments of the relationships between these patients' three major impairments and supporting their efforts to find effective coping approaches while doing so are critical.

In developing coping techniques, the participants in this study acted independently in various ways, including <planning the timing of their bowel movements $\rangle$, 〈planning their meals $\rangle$, 〈keeping their abdomen warm $\rangle$, 〈changing their routines $\rangle$, 〈planning their outdoor activities〉, and 〈resisting bowel movement urges〉. Based on the advice of their doctors and nurses, participants also performed coping measures related to their nursing and treatment interventions, such as <strengthening the anal sphincter to improve soiling > and 〈regulating bowel movements with drug therapy $\rangle$. Prior studies have also discussed the effectiveness of these measures, but as differences in effectiveness were observed between patients, ${ }^{18}$ the appropriate timing for providing information of suitable quality and quantity as well as the method of providing support must be evaluated in order to ascertain participant readiness.

Based on the process characterized by this study, it is necessary to develop a support program structured based on the level of patients' impairment and their level of readiness. Additionally, developing an expert 
care service for outpatients such as a consultation program as well as organizing a system or framework to enable expert nurses to provide support to ensure that the best possible supportive care is provided to male rectal cancer patients even after discharge are also desirable. It is also necessary to establish a collaborative support structure that extends beyond the hospital to include patient advocacy organizations as well.

\section{Limitations and recommendations}

This study was not designed to be widely generalizable, as it targeted male rectal cancer outpatients undergoing surgery at the same facility. Additionally, it is possible that differences in surgical procedures and postoperative periods may have had some impact on results.

In the future, we would like to assess differences based on surgical procedure used, length of postoperative period, and individual patient background traits such as sex and age group. Including not just a single facility but multiple clinics in different locations is also necessary to broaden assessment.

\section{Acknowledgments}

We would like to extend our sincere gratitude to the medical facility staff and the numerous patients who agreed to participate in this study.

\section{Conflicts of interest statement}

There was no conflicts of interest to disclose with respect to this study.

\section{References}

1. Cancer Information Service Cancer Statistics in Japan-2015, http://ganjoho.jp/reg_stat/statistics/stat/index.html.2016. 3.1.

2. Takahashi K, Matsumoto H, Yamaguchi T, et al. Surgery for low rectal cancer based on QOL. Geka Chiryo (Surgical Therapy) 2009; 101(4): 472-478 (Abstract in English).

3. Itabshi M, Hirosawa T, Ogawa S, et al. Surgical treatment for colorectal cancer. Daityogan Frontier (Frontiers in Colorectal Cancer) 2008; 1(1): 26-29 (Abstract in English).

4. Sato M. Development of "Defecation Dysfunction Assessment Scale ver. 2" for evaluation after anterior resection for rectal cancer. Nippon Stoma Haisetsu Rehabilitation Gakkai (Journal of Japanese Society of Stoma and Continence Rehabilitation) 2010; 26(3): 37-48 (Abstract in English).

5. Tsuji A, Suzuki Y. Psychological after effects of defecation dysfunction on patients with LAR and their strategies. Nippon Igaku Kangogaku Kyouiku Gakkaishi (Japanese Journal of Medical and Nursing Education) 2009; 18: 34-38
(Abstract in English).

6. Tsuji A, Suzuki Y, Yamaguchi T, et al. Actual situations of defective dysfunction after low anterior resection and instructions to patients. Wakayamakenritsu Ikadaigaku Hoken Kangogakubu Kiyou (Bulletin of School of Health and Nursing Science, Wakayama Medical University) 2007; 3: 5-15 (Abstract in English).

7. Au TY, Zauszniewski JA, King TM. Demographics, cancer-related factors, and sexual function in rectal cancer patients in Taiwan: preliminary findings. Cancer Nurs 2012; 35(5): 17-25.

8. Liu $\mathrm{CH}$, Chen $\mathrm{CH}$, Lee JC. Rehabilitation exercise on the quality of life in anal sphincter-preserving surgery. Hepatogastroenterology 2011; 58: 110-111.

9. Landers M, McCarthy G, Savage E. Bowel symptom experiences and management following sphincter saving surgery for rectal cancer: a qualitative perspective. Eur J Oncol Nurs 2012; 16(3): 293-300.

10. Beech N, Arber A, Faithfull S. Restoring a sense of wellness following colorectal cancer: a grounded theory. J Adv Nurs 2012; 68(5): 1134-1144.

11. Strauss AL, Corbin J. Grounded theory methodology: an overview. In: Strategies of Qualitative Inquiry. Thousand Oaks: Sage, 1998: 158-183.

12. Blumer, H.G. Symbolic Interactionism: Perspective and Method, Prentice-Hall: Englewood Cliffs, N. J., 1969: 20-21.

13. Yumiko K, Akiko C, Rieko K, et al. A longitudinal study of gender differences in quality of life among Japanese patients with lower rectal cancer treated with sphinctersaving surgery: a 1-year follow-up. World J Surg Oncol 2015; 13: 91-105.

14. Takehara S, Take A, Nishizono M, et al. Coping strategies among patients experiencing defecation dysfunction following closure of a temporary stoma due to a low anterior resection for rectal cancer. Nippon sousho Ostomy Shikkin Kanri Gakkai (Journal of Japanese Society of Wound, Ostomy and Continence Management) 2015; 19(4): 386-393 (Abstract in English).

15. Desnoo L, Faithfull S. A qualitative study of anterior resection syndrome: the experiences of cancer survivors who have undergone resection surgery. Eur J Cancer Care 2006; 15(3): 244-251

16. Maeda M, Oishi F, Hayama Y. The process of life reconstruction in rectal cancer patients after total pelvic exenteration. Nippon Gan Kango Gakkaishi (Journal of Japanese Society of Cancer Nursing) 2012; 26(2): 6-16 (Abstract in English).

17. Fujita A, Sato W, Oka M, et al. The relationship between postoperative bowel dysfunction and self-esteem in the postoperative course period after low anterior resection for rectal cancer patients. Nippon Kango Kagaku Gakkaishi (J Jpn Acad Nurs Sci) 2002; 22(2): 34-43 (Abstract in English).

18. Sato M, Hidaka K. Review of the nursing care for rectal cancer patients with defecation disorder following anterior resection. Nippon Kango Kagaku Gakkaishi (J Jpn Acad Nurs Sci) 2012; 32(2): 64-71 (Abstract in English). 